\title{
NOTAS A PROPÓSITO DAS BLASTOMICOSES PROPRIAMENTE DITAS
}

FLORIANO DE ALMEIDA

$\left(1 .^{\circ}\right.$ assistente e docente-livre)
CARLOS DA SILVA IACAZ

(2.0 assistente substituto)

Numerosas formas clínicas encontram-se rotuladas com a denominação genérica de blastomicose. Abusivamente tal nome tem sido empregado, razão pela qual o conceito exato das blastomicoses precisa ser convenientemente estabelecido.

A classificação das blastomicoses proposta por Flavio Niño (1) do ponto de vista médico é utilíssima e pode ser assim esquematisada:

1. ${ }^{\circ}$ grupo - Blastomicoses propriamente ditas:

Estomatite cremosa ou sapinho

Oníxis e perioníxis blastomicéticas

Vulvovaginites blastomicéticas

Intertrigos blastomicéticos

Septicopiohemia blastomicética

Queratites blastomicéticas

Dermatites blastomicéticas
Produzidas por cogumelos pertencentes aos gêneros: Saccharomyces, Willia, Mycotorula, Mycotoruloides, Candida, Mycocandida, Blastodendrion, Geotrichum, Néogeotrichum, Proteomyces etc.

Granuloma criptocócico $\left\{\begin{array}{l}\text { Formas generalisadas } \\ \text { Formas localisadas }\end{array}\left\{\begin{array}{l}\text { Produzido pelo Cry- } \\ \text { ptococcus neoformans. }\end{array}\right.\right.$

2. grupo - Parablastomicoses:

a) Granuloma paracoccidioidico ... Paracoccidioides brasiliensis

b) Sindrome de Gilchrist ....... Zymonema dermatitidis

c) Histoplasmose ........... Histoplasma capsulatum

3. grupo - Pseudoblastomicoses:
a) Granuloma coccidióidico ......
b) Granuloma rinosporidiósico ....
c) Granuloma cromomicósico ....
Coccidioides immitis
Rhinosporidium Seeberi
Vários gêneros.

(1) Fravio L. Niño - Contribución al estudio de las blastomicosis en la República Argentina (Boletin del Instituto de Clinica Quirurgica - Julio de 1938 - Buenos Aires). 
As blastomicoses propriamente ditas são produzidas por leveduras, verdadeiras ou falsas, reproduzindo-se nos tecidos por um brotamento ou gemulação. As parablastomicoses são determinadas por cogumelos que sem ser leveduras podem em algumas circunstâncias se apresentar ao exame microscópico como células gemulantes que recordam em sua morfologia as leveduras verdadeiras. As pseudoblastomicoses são produzidas por fungos que em nenhum momento apresentam a forma de reprođução por brotamento ou gemulação.

As verdadeiras blastomicoses são na realidade, as determinadas por leveduras, verdadeiras ou falsas. 'No entanto, entre nós, generalisou-se para o granuloma paracoccidioídico a denominação de "blastomicose brasileira". Os A.A. norte americanos e italianos (2-3-4-5) reservam para esta entidade clínica a denominação de moléstia de Lutz-Splendore-Almeida ou moléstia de Almeida, como quer WEIDMANN.

De um modo geral as verdadeiras blastomicoses são sensiveis ao tratamento iódico intensivo, ao passo que o granuloma paracoccidioidico raríssimas vezes responde bem à iodoterapia.

Vemos pois que do ponto de vista terapêutico há enorme importância em se estabelecer com precisão um diagnóstico etiológico exato.

ReDAelli (6) separa, aliás, as blastomicoses em 2 grupos distintos: $1^{\circ}$ ) aquelas produzidas por levedurảs ascógenas ou anascógenas e $2 .^{\circ}$ ) aquelas produzidas por fungos que encerram a maior parte das chamadas blastomicoses americanas.

Analisāndo neste trabalho somente as blastomicoses propriamente ditas de acordo com a classificação proposta por Flavio Niño, referiremos alguns dados por nós obtidos na Secção de Micologia da Faculdade de Medicina.

A estomatite cremosa conhecida com o nome de "sapinho bucal" ou "muguet" é uma blastomicose relativamente benigna e só terá valôr prognóstico quando aparecer nos indivíduos em idade avançada, nos diabéticos e nos caquéticos de um modo geral. $O$ assunto mereceu um estudo cuidadoso por parte de Inah Moraes de Camargo (7)

2) R. Cifrerri e P. Redaeli - Paracoccidioideaceae, N. Fam., instituida per l'agente del "granuloma Paracoccidioide" (Paracoccidioides brasiliensis) (Bollettino dell'Instituto Sieroterapico Milaneze - Vol. XV - Fasc. II - Febbraio 1936 - Pagine 97-102).

(3) Piero Redaeldi e Rafaele Ciferri - Morfologia, biologia e posizione sistematica di Paracoccidioides brasiliensis (Splendore) Almeida (Fam. Paracoccidioideaceae) con notizie sul granuloma paracoccidioide (Reàle Academia d'Italia - Memorie della Classe di Scienze Fisiche, Matematiche e Naturali - Vol. VIII - Estratto n. 12 - 1937 -
Roma).

(4) MORris Moore - Blastomycosis, Coccidioidal Granuloma and Paracoccidioidal Granuloma (Archives of Dermatology and Syphilology - August 1938 - Vol. 38 - pp.
163-190).

(5) T. W. JoRdan and T. O. WeidmanN - Coccidioidal Granuloma. Comparison of the North and South American diseases with special reference to Paracoccidioides brasiliens (Arch. Dermat. and Syphilol. \$3-31-1936).

(6) P. REDAELLI - L'attuale sistemazione delle cosidette "Blastomicosi" (Estratto dell'Instituto Biochimico Italiano - n.0 10 - Anno XIV - 1936).

(7) Inat Moraes de Camargo - Agentes etiologicos do "sapinho", estomatite cre. mosa em São Paulo. Tése. São Paulo. 1934. 
que defendeu sua tése de doutoramento sobre o sapinho, estudando a sua etiologia, patologia e tratamento.

Uma questão interessante relacionada ao sapinho bucal é a da vulvo-vaginite blastomicética, muito comum nas mulheres em gestação. Este assunto já foi por nós estudado (8-9 e 10), parecendo haver uma estreita relação entre o sapinho vaginal e o sapinho bucal dos recém-nascidos; estes 'se contaminariam durante sua passagem pelo canal vaginal onde se encontraríam os cogumelos agentes do sapinho. Negroni, na Argentina, acredita que 33\% das mulheres grávidas apresentam o sapinho vaginal nos seus diferentes aspectors clínicos.

Woodruff e Hessetine (resumo de um trabalho publicado no Boletim de la Of́icina Sanitária Panamericana, Año 18-n. ${ }^{\circ}$ 3-Marzo de 1939) realizaram cultivos vaginais de 402 mulheres no $3 .^{\circ}$ trimestre da gravidez e estudaram 90 casos de sapinho bucal dos recémnascidos. $\mathrm{O}$ coeficiente da micose vaginal segundo aqueles autores parece associar-se com a situação econômica e higiênica das mulheres, correnda os recém-nascidos dessas mulheres 35 vezes mais 'perigo de manifestar o sapinho que as filhos de mães indenes de infecção. A frequência do sapinho vaginal naquelas mulheres foi de $28 \%$; as indigentes pretas apresentavam-se com o sapinho vaginal em maior proporção que as indigentes brancas e as brancas mais ilustradas quasi não eram atacadas pela micose vaginal. Claúdius $P$ Jones e DoNALD S. MARTin (11) estudaram 68 amostras de cogumelos leveduriformes isolados do trato vaginal de mulheres grávidas e não grávidas, sendo que dessas amostras 52 foram isoladas das primeiras e 16 das segundas. Vemos pois que a porcentagem de cogumelos isolados da vagina de mulheres grávidas tem sido apreciavel e este fato pode explicar até certo ponto o sapinho bucal dos recémnascidos. Uma outra fonte de origem do sapinho bucal dos recémnascidos talvez seja representada pelas rágades ou rachaduras dos bicos dos seios maternos que surgem logo após o parto.

Glossites blastomicéticas nós já as temos observado e em 1939 publicamos (12) um interessànte caso de glossite superficial membranosa provocada por um cogumelo do gênero Candida. Casos de "lingua negra pilosa" foram por nós observados 3 vezes, tendo sido

(8) Floriano de almeida e Carlos da Silva lacaz - Considerações em torno do sapinho vaginal e bucal (Rev. de Obstetrícia e Ginecologia de São Paulo - Vol. III -Fasc. I - 1938).

(9) Carlos dA S. Lacaz - Sapinho vaginal (Medicina Pratica - Nova Era - Ano I - n. 2 - julho de 1938).

(10) Cartos dA S. Lacaz - Sapinho vaginal (Com. Dep. Cientifico do CAOC Sessão de 23/5/1938).

(11) Claudius P. Jones and Donald S. Martin - Identification of yeastlike organisms isolated from the vaginal tracts of pregnant and nonpregnant women (Am. Journ. of Obstetrics and Gynecology - vol. 35 - n. 1 - Page 98 - January 1938).

(12) Floriano de Almeida e Carios da S. Lacaz - Consideraçóes sobre um caso de glossite superficial membranosa de natưreza micótica (Folia Clinica et Biologica - Vol. XI -1939 - n. ${ }^{\circ} 1$ - São Paulo). 
isoladas em cultura pura 3 leveduras. Ambos os pacientes fumavam em excesso e em um deles somente a abolição desse vício curou-o radicalmente.

Recentemente, Cotrins (13) publicou um caso de língua negra pilosa determinado pela Mycotorula Guilliermondi (Cast.) n. comb. e o doente curou-se completamente com o emprego intensivo de vitamina A.

Nesses casos o cogumelo secundariamente se instala sobre a mucosa lingual previamente alterada por uma causa tóxica (fumo) ou por um deficit de vitamina A que é a vitamina protetora dos epitélios.

Cogumelos leveduriformes foram igualmente por nós isolados dè lesões das comissuras labiais, conhecidas vulgarmente com o nome de "boquera".

Nesses casos a radioterapia deu ótimos resultados terapêtuticos, pois ta1s lesões resistem à ação do iodo.

Tivemos ocasião de observar um caso de faringite aguda blastom micética em um japonês que tinha o diagnóstico de "blastomicose brasileira".

Um exame cuidadoso do material retirado das lesões revelou inúmeros filamentos micelianos e células leveduriformes com ausência do Paracoccidioides brasiliensis. A cultura foi positiva para um cogunelo do gênero Candida. Terapêutica iódica altamente eficás.

Este caso mostra mais uma vez a vantagem de se estabelecer com precisão um diagnóstico etiológico exato.

As figuras 1 e 2 mostram um caso de glossite e. les̃ões da mucosa labial determinadas por um cogumelo leveduriforme. O diagnóstico uma vez estabelecido, o doente curou-se radicalmente e em pouco tempo com a medicação iódica intensiva.

A figura 3 nos mostra um outro doente com lesões ulcerativas da mucosa do lábio inferior e dos quais isolamos um cogumelo pertencente ao gênero Candida. A figura 4 mostra um aspecto da colônia do cogumelo isolado.

Em cultura pura já isolamos (14) da bile C 4 tipos de cogumelos leveduriformes em pacientes com sintomatologia hépato biliar. Em um caso de retite ulcerativa isolamos (15) uma levedura per. tencente ao gênero Geotrichum.

Lesões de perionixis determinadas por cogumelos leveduriformes são comuns em nosso meio. As oníxis são ao contrário, geralmente

(13) G. B. Cottrin - Un caso di "lingua nigra et pilosa" con isolamento di Ḿycothrula Guilliermondi. (Cast.) n. comb. (Mycopathologia - Vol. II - 30. VII - 1939
- Fasc. 2).

(14) Floriano de Almeida e Carlos da S. Lacaz - Cogumelos levediformes isolados da bile Folia et Biologica - n. ${ }^{\circ} 3$ - Vol. XII - 1940).

(15) Floriano de Almeida e Carlos da S. LACAz - Cogumelo do gênero Geotrichum isolado de lesões ulcerativas do reto Folia Clinica et Biologica - Vol. XII - n.o $2-$ 


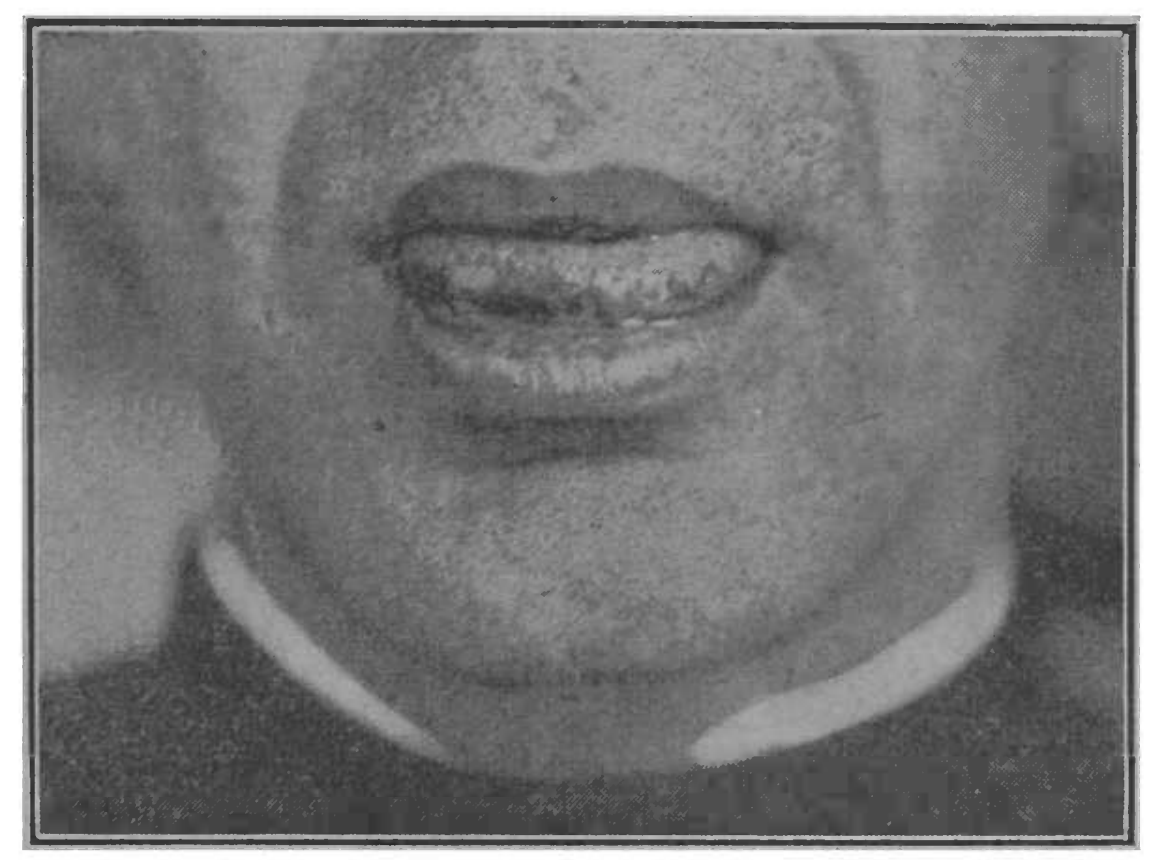

FIG. N. ${ }^{\circ} 1$

Doente M.G.B. com glossite ulcerativa blastomicética.

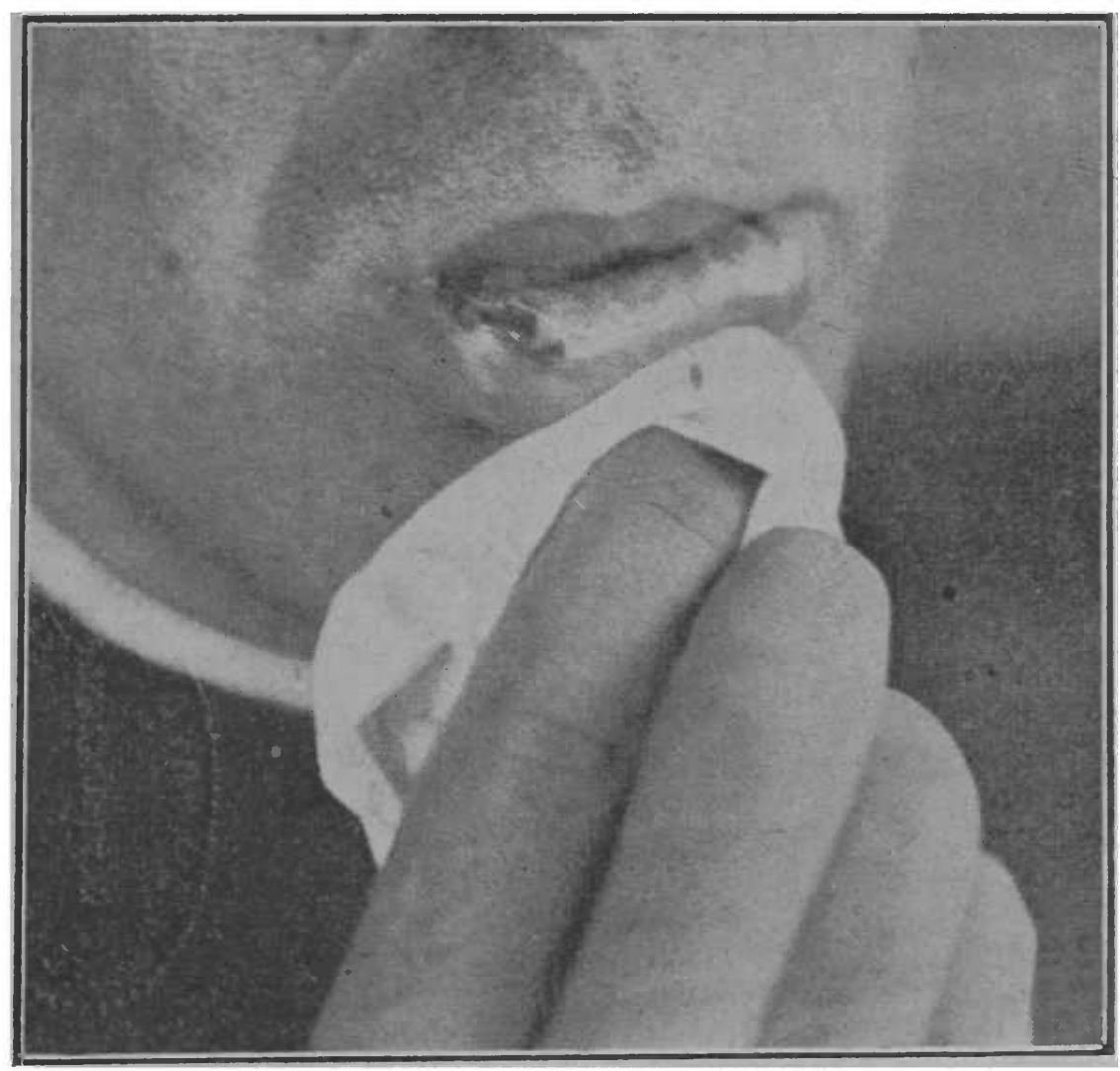

Fig. x.a 2

Doente M.G.B. mostrando as lesões de estomatite ulcerativa. 
determinadas por cogumelos da tribu Trichophytoncac. (asos desta natureza já tivemos (16) ocasião de assinalar em trabalho publicado nos Arquivos de Cirurgia Clínica e Experimental.

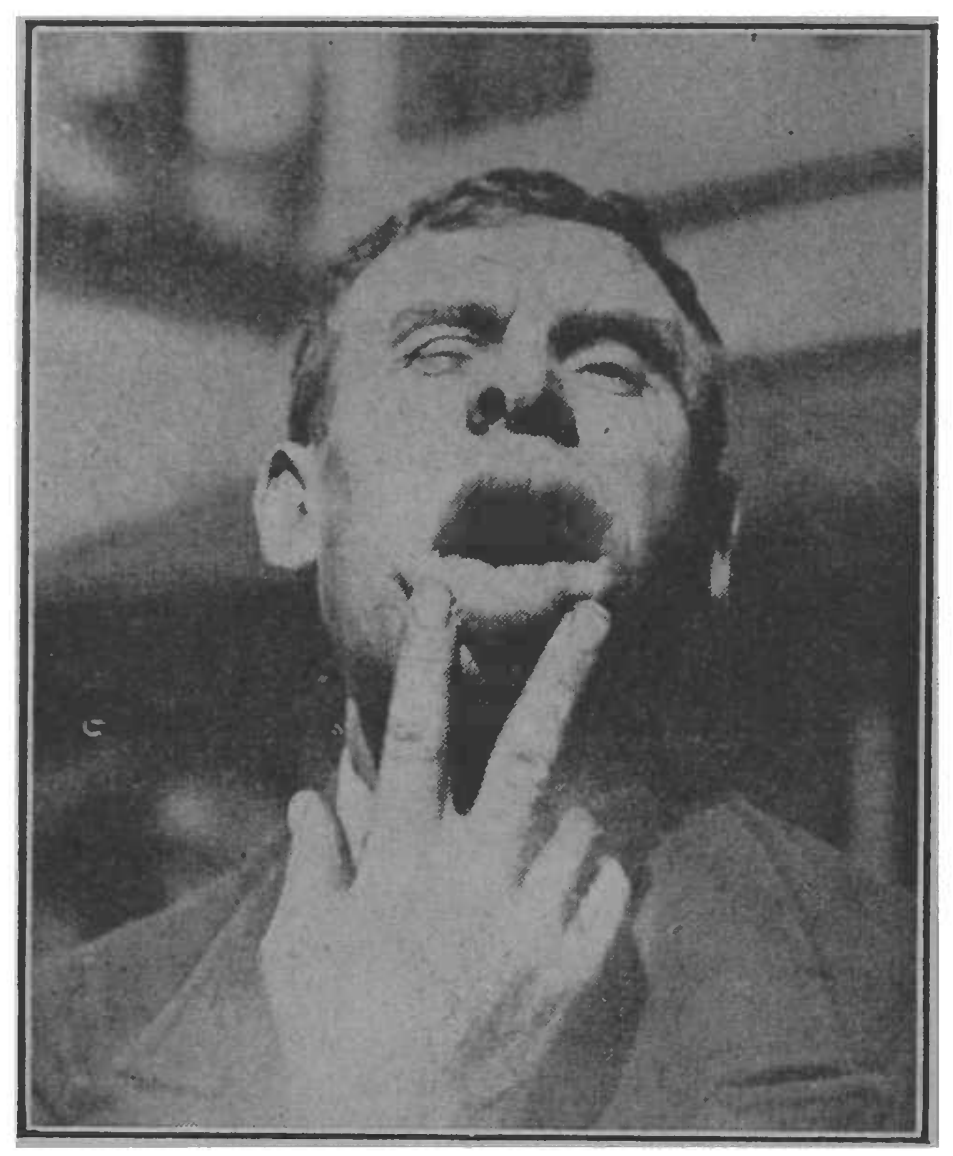

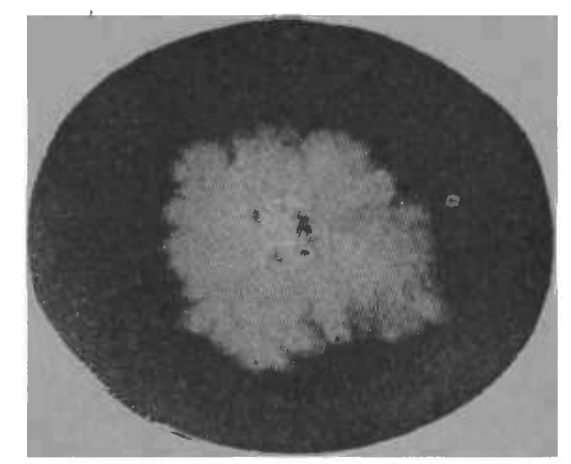

FIG. N, ${ }^{\circ} 4$

Colonia gigante em Sabouraud glicose do cogumelo isolado do caso anterior.

Caso B. - Estomatite blastomicética.

Dermatites blastomicéticas não devem ser raras em nosso meio, mas infelizmente não receḅemos material abundante para estudo. Apenas um caso desta natureza nos foi enviado pelo Prof. Aguiar Pupo e sobre ele já publicamos (17) um trabalho nos Anais Paulistas de Med. e Cir.

Quanto às micoses pulmonares por leveduras elas são muito frequentes, podendo se apresentar primitivas, secundárias ou associadas. Pretendemos em breve publicar um trabalho sobre este assunto encarando a necessidade do seu estudo, debaixo de um ponto de vista criterioso.

(16) Floriano de Almeida e Carlos Da S. Lacaz - Considerações em torno das micoses cirurgicas (Arquivos de Cirurgia Clinica e Exp. - Vol. III - n. ${ }^{\circ} 2$ - abril
de 1939).

(17) Floriano de Almeida e Carlos da S. Lacaz - Considerações sobre um caso de blastomicose cutaneo-mucosa (Anais Paulistas de Med. e Cir. - Vol. XXXVIII - out.
1939 - n. 4). 


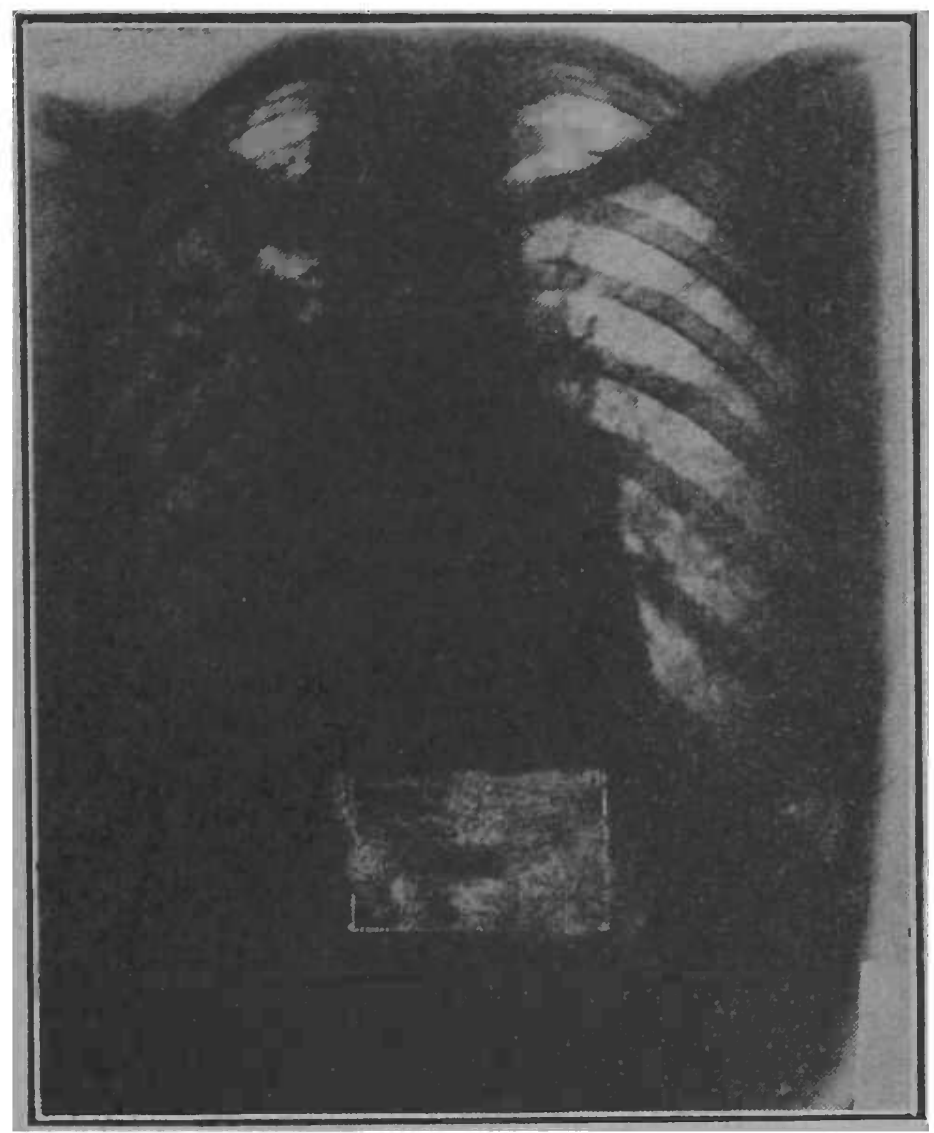

FIG. N. ${ }^{\circ} 5$

Broncopulmonite blastomicética. Doente Y. F. antes do tratamento.

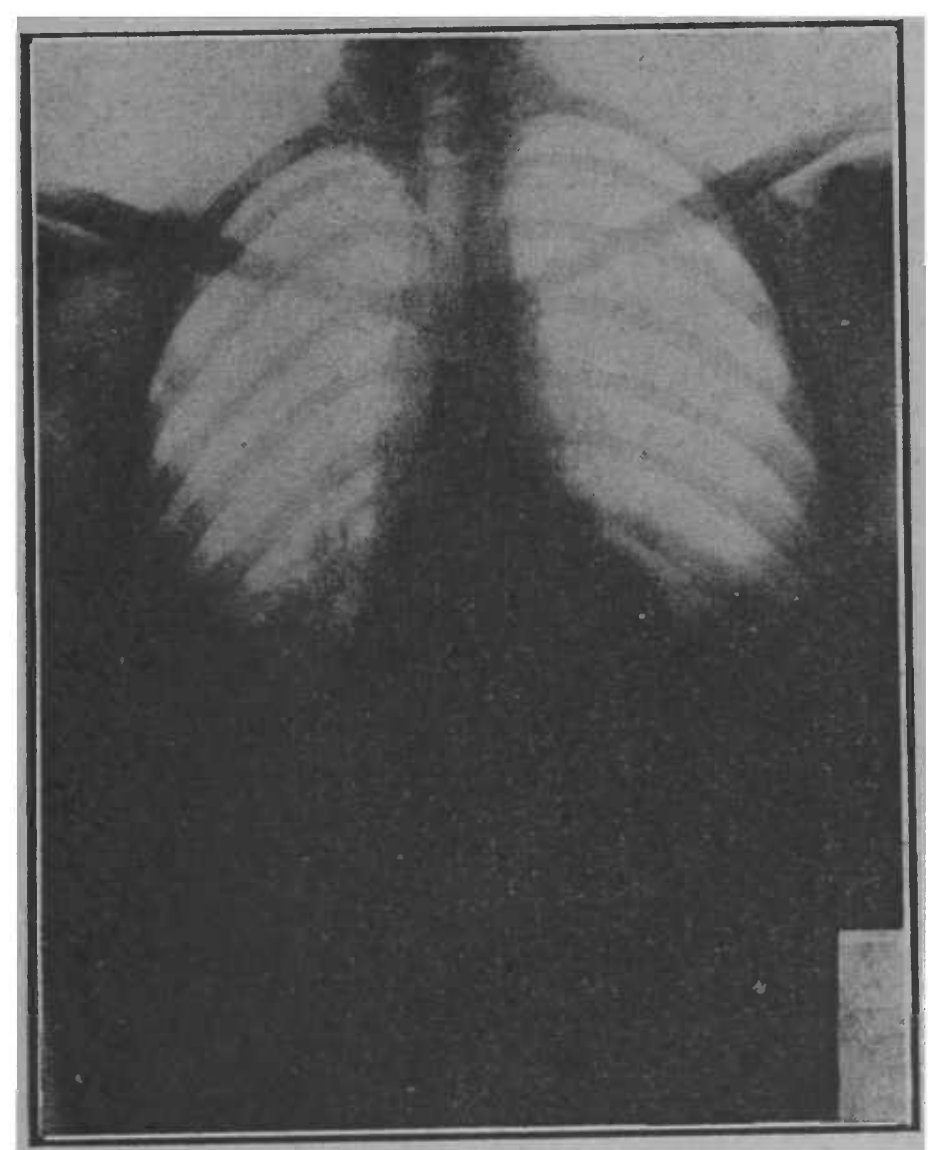

FIG. $\times \circ 6$

Doente Y. F. após o tratamento. 


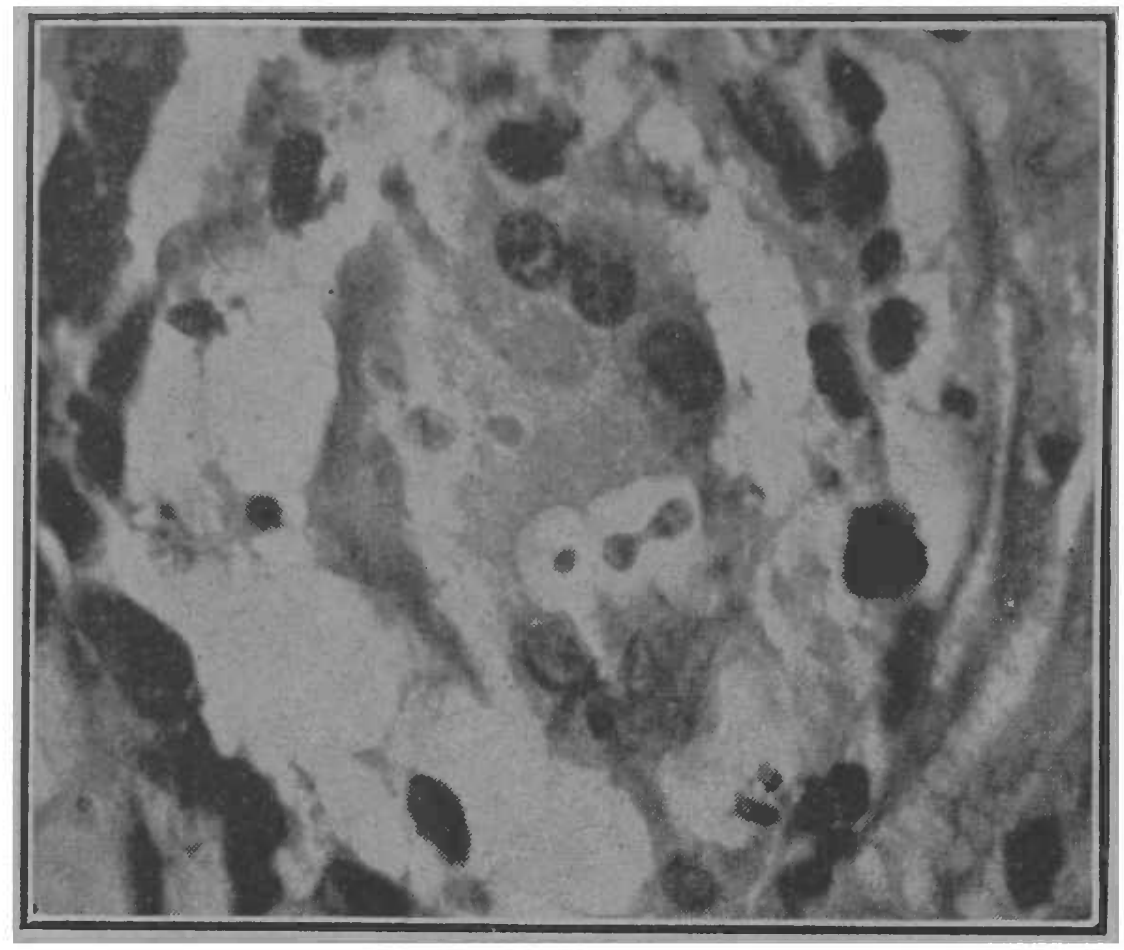

FrG. N. ${ }^{7} 7$

Cogumelos leveduriformes no tecido. Caso cie dermatite blastomicética. Doente A. A.

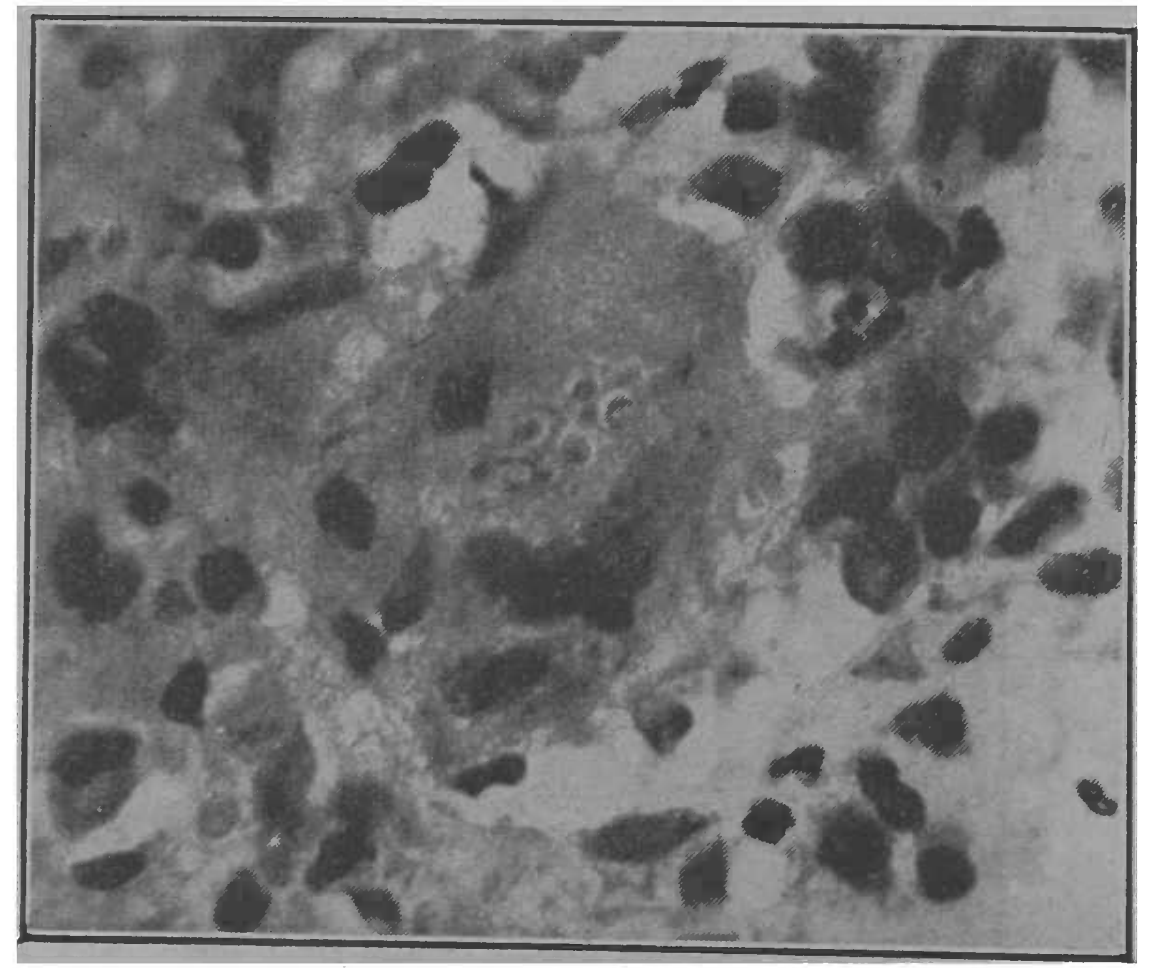

FIG. N.0 8

Cogumelos leveduriformes no tecido. Caso de dermatite blastomicética. Doente A. A. 
Em várias notas publicadas temos chamado (18-19-20-21-22-2324-25-26) a atenção dos clínicos em geral para as broncopulmonites blastomicéticas, encarando o diagnóstico dessas formas clínicas, a sua etiopatogenía e terapêutica.

A sintomatologia apresentada por esses doentes é em tudo idênt.ca a da tuberculose e somente o laboratório elucidará a etiologia do processo.

Queremos nos referir finalmente ao granuloma criptocócico determinado pelo Cryptococcus neoformans (SANFELICE) Vüillemin 1901.

A moléstia determinada por este cogumelo apresenta quadros clínicos os mais variados, predominando porém as lesões cerebrais; pulmonares, cutâneas e ósseas.

Uim caso desta natureza já foi por nós obsérvado no ponto de vista micológico. O material nos foi enviado pelo IDr. FAứsto D'Oliveira Quaglia, de Rio Preto, que isolou o fungo diretamente do líquido céfalo raquidiano. Grordano (27), Redaelli e Ciferri (28) dão ao fungo a denominação de Debaryomyces neoformans (SANFELICE) Red., Cif. “e Giordano 1937.

Diagnóstico: o diagnóstico preciso de uma blastomicose propriamente dita repousa em $1 .^{\circ}$ lugar na observação microscópica do fungo no material retirado das lesões. O exame microscópico poderá ser feito pelo método de Gram ou ZieHL e nos casos positivos verificaremos numerosas células gemulantes, esféricas ou ovóides, ao lado de filamentos micelianos em maior ou menor abundância.

No caso particular do Cryptococcus neoformans o exame direto nos revela numerosos cogumelos de forma arredondada ou ligeira-

(18) Floriano de Almeida e Carlos da S. LAcaz - Conśiderações micológicas sobre 6 amostras de lêvedos isoladas do escarro An. Fac. Med. S. Paulo - Voḷ. XVI - 1940).

(19) Floriano de Almeida e Carlos da S. LACAZ - Cogumelo do gênero Saccharomyces isolado do escarro (An. Fac. Med. S. Paulo - Vol. XVI - 1940).

(20) Floriano de Almeida e Carlos da S. Lacaz - Processos pulmonares míxtos com especial referencia à associação tuberculo micótica (An. Paul. Med. e Cir. - maio de 1940).

(21) Floriano de Almeida e Carlos da S. Lacaz - Considerações micológicas sobre 4 amostras de Geotrichum isoladas do escarro (Folia Clinica et Biologica — Vol. XII — n. ${ }^{\circ}$ 2 - 1940).

(22) Floriano de Almeida e Carlos da S. Lacaz - Frequencia das micoses pulmónares em São Paulo Rev. Med. CAOC - vol. 23 - junho de 1939 - n.o 66).

(23) Floriano de Almeida - Cons. sobre as micoses pulmonares (Conf. Sessão Tisiologia da Assoc. Paulista de Med. - 23/5/1941).

(24) Floriano DE Almeida - Mycoses do aparelho respiratorio (Anais Paul. Med. e Cir. - Vol. XXX - n. ${ }^{\circ} 6$ - Dez. 1935).

(25) Floriano de Almeida - Cons. sobre micoses pulmonares (Boletim Soc. Med. e Cir. - São Paulo, vol. XVIII - 1933 1934).

(26) Carios Da S. Lacaz - Pneumomicoses (Rev. Clin. São Paulo - vol. IV -.. n.o $1-18-32)$.

(27) A. Grordano - Studio micologico del "Debaryomyces neoformans (Sanfelice) Red., Cif. et Giordano e significato della specie nella patologia animale Mycopathologia - vol. I - 21-1-1939 - Fasc. 4).

(28) P. REDaElit, R. CifERri e A. Giordano - Debaryomyces neoformans (Sanfelice nobis, $n$. comb. pour les espèces du groupe Saccharomyces hominis (Bollettino della Sezione Italiana della Società Internazionale di Microbiologia - Fasc. I-II - GennaioFebbraio 1937). 
mente ovóide, providos de uma dupla membrana e rodeados por uma cápsula de aspecto mucoso ou gelatinoso, cápsula esta que confere ao parasito um aspeto característico. No citoplasma desse cogumelo observam-se gotículas de gordura misturadas a uma substância granulosa, às vezes rica em cromatina.

Outras vezes, a observação do fungo é feita nos tecidos, aparecendo o cogumelo com o seu aspecto característico de leveduras (figuras 7 e 8 ).

A reação histopatológica é do tipo granulomatoso.

Observado o cogumelo resta o seu isolamento e identificação. Para o isolamento usamos preferencialmente a gelose glicosada e acidificada pelo ácido tartárico a $2 \%$, meio este disposto em placas de Petri.

A identificação da levedura obedece ao seguinte critério por nós adotado:

a) estudo macroscópico da colônia gigante do cogumelo.

b) estudo micromorfológico, do fungo em água de batata, segundo o processo de LANGeron e TALice (29) ou em água de fécula de batata segundo nossas observações (30).

c) estudo bioquímico.

d) crescimento do cogumelo em mosto gelàtinado.

Para um diagnóstico genérico grosseiro do cogumelo isolado bastam esses exames e de acordo com o quadro seguinte, consegue o analista identificar a amostra isolada com relativa facilidade.

Uma vez identificado o cogumelo devemos praticar a sua inoculação em animaís sensiveis, tendo-se o cuidado de sensibilizar o animal anteriormente para que os resultados sejam positivos, conforme assinala HENRICI (31). A intradermo reação com o extrato do cogumelo isolado, a soro aglutinação e a reação de fixação de complemento são exames subsidiários de valor. Para maiores detalhes sobre tais exames consulte-se os trabalhos de Cavallero (3233) e o livro de M. Lewis e Mary Hopper (34).

(29) M. Langeron et R. V. TALice - Nouvelles méthodes d'étude et éssai de classification des champignons levuriformes (Annales de Parasitologie humaine et comparée -

(30) Floriano de Almeida e Carlos da S. Lacaz - Nóva tecnica para demonstração rapida des ascosporos (Folia Clinica et Biologica - vol. $12-1940-\mathbf{n . 0}^{\circ} 4$ ). (31) ARThur T. HENRICI - Characteristics of Fungous Díseases (Journal of Bacte-
riology - Vol. $39-$ n.0 $2-$ February 1941).

(32) Cavallero - L'allergia e l'immunità nelle micosi. 1.': Concetti generali sull'al8.V.1940 - Fasc. 4 e Vol immunità nelle micosi da lieviti (Mycopathologia - Vol. II (33) CAvar. 4 e Vol. III - 10-II-1941 - Fasc. 1) nele micosi da lieviti (Blastomicosi) Mycopathologia nelle micosi. 2.0 Allergia e immunità (34) George M. Lewis AND Mary E

Chicago, Illinois. 1939. 


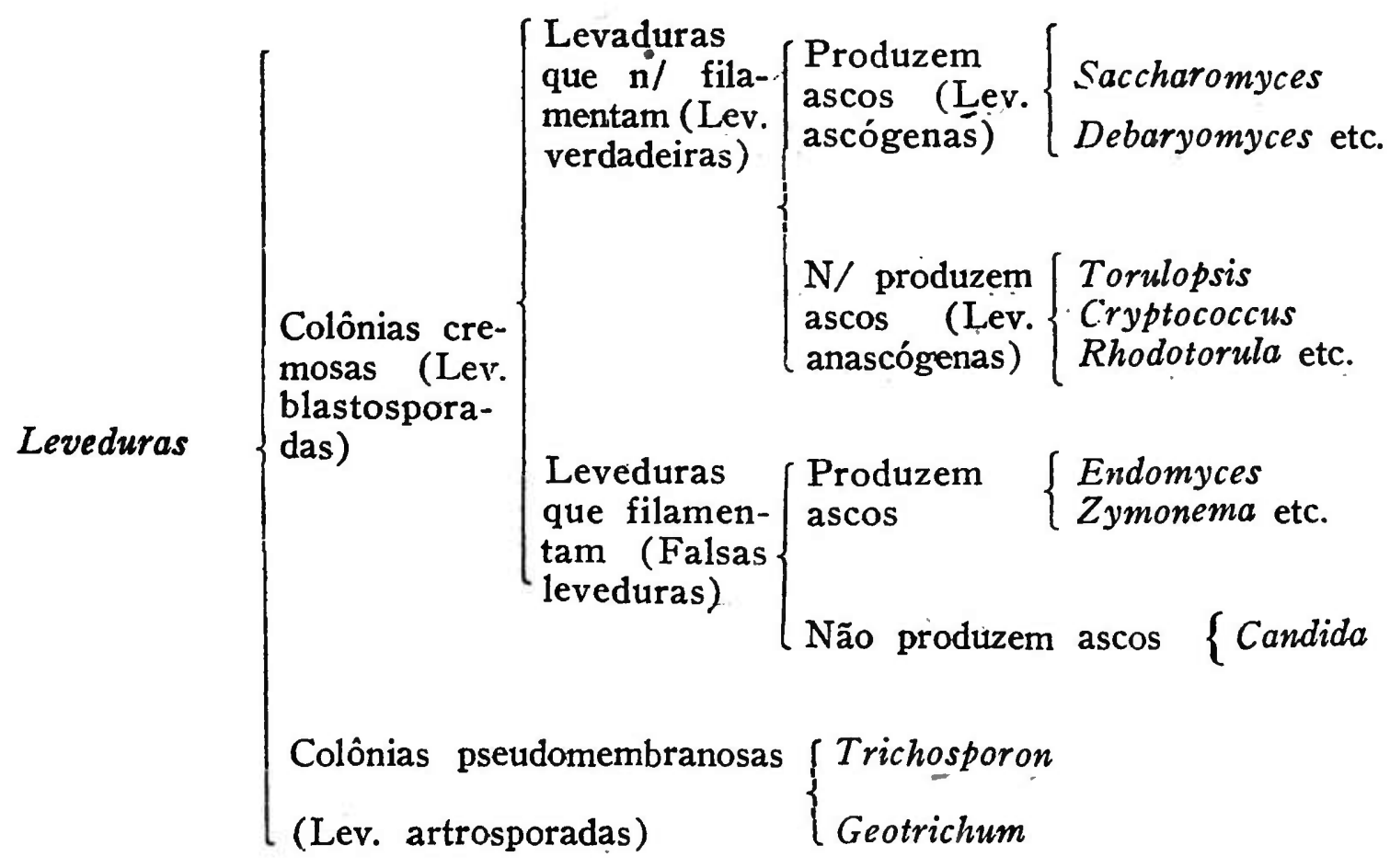

Tratamento: o tratamento das blastomicoses propriamente ditas, a não ser nos casos de granuloma criptocócico, oferece bons resultados quando é bem conduzido. A iodoterapia intensa oferece resultados satisfatórios na maioria dos casos, particularmente nas broncopulmonites blastomicéticas e dermatites. A vacinoterapia pode ser empregada nos casos rebeldes, assim com a sulfamidoterapia.

Nas perionixis blastomicéticas é aconselhavel a radioterapia porque o tratamento médico local não oferece resultados.

No sapinho, quér vaginal, quér bucal, o emprego de solutos alcalinas, localmente, dá ótimos resultados a não ser que tais micoses apareçam nos diabéticos e nos caquéticos.

No caso espec:al da micose determinada pelo Cryptococtus neoformans o prognóstico da moléstia é gravíssico, porque as lesões se generalisam. Niño mostrou que a solução de YATREN a $5 \%$ em soro fisiológico assim como o corpo 386 da Casa Bayer exercem in vitro uma ação inibidora sobre este parasito. Com estes medicamentos conseguiu curar um caso de granuloma criptocócico. 


\section{P Y
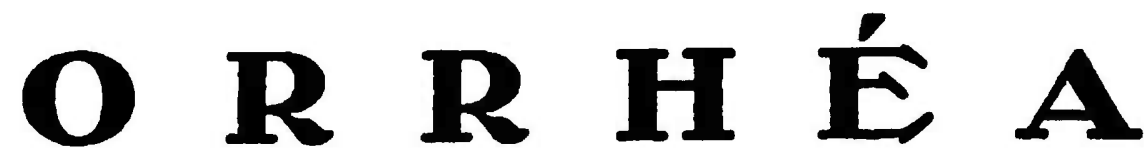

Gengivas sangrentas, dentes abalados e mau halito:

Resultados positivos em 8 dias, com o especifico

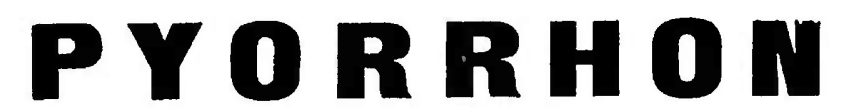

CONSULTAS: $30 \$ 000$.

DEMONSTRAÇõES PRATICAS AOS SENHORES MEDICOS E DENTISTAS

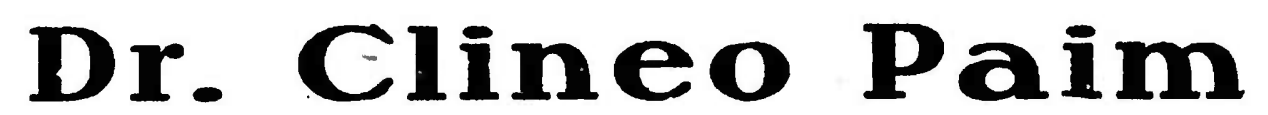

R. Barão de Itapetịninga, $120-5 .^{\circ}$ andar - Salas, 505 e 506 (CASA GUATAPARÁ)

TELEFONE: 4-4050 - SÃO PAULO

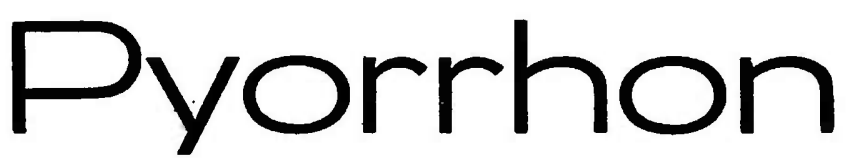

Um medicamento que veio .resolver os casos de Gengivites e Prornéa

\section{A T E S T A D O}

E' para mim um prazer atestar que venho empregando em minha clinica com os mais brilhantes resultados, o Pyorrhon, medicamento de escol para o tratamento da Piorrhéa Alveolar e das Gengivites.

Tambem venho calorosamente recomendando o seu uso aos meus pacientes, porque assim fazendo estes teem assegurada a perfeita saude do seu meio bucal.

o Pyorrhon é um preparado que pela propaganda honesta com que é lançado e pelos seus meritos, merece da nossa classe a melhor acolhida.

São Paulo, 6 de Outubro de 1939.

Octavio Demacq Rosas.

\section{Receite PYORRHON aos seus clientes}

